\title{
An Effective Fully Polarizable QM/MM Approach to Model Vibrational Circular Dichroism Spectra of Systems in Aqueous Solution
}

\author{
Tommaso Giovannini, ${ }^{\dagger}$ Marta Olszòwka, ${ }^{\ddagger}$ and Chiara Cappelli ${ }^{*, \dagger}$
}

Scuola Normale Superiore, Piazza dei Cavalieri 7, 56126 Pisa, Italy, and Dipartimento di

Chimica e Chimica Industriale, Università di Pisa, Via Moruzzi 3, 56124 Pisa, Italy.

E-mail: chiara.cappelli@sns.it

${ }^{*}$ To whom correspondence should be addressed

${ }^{\dagger}$ Scuola Normale Superiore, Piazza dei Cavalieri 7, 56126 Pisa, Italy

${ }_{\ddagger}^{\ddagger}$ Dipartimento di Chimica e Chimica Industriale, Università di Pisa, Via Moruzzi 3, 56124 Pisa, Italy. 


\section{S1 (R)-Methyloxirane}

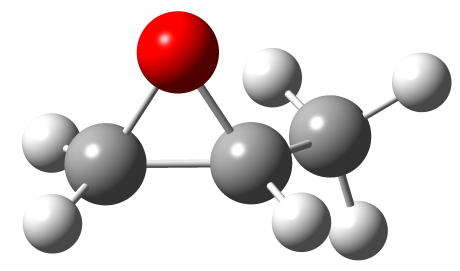

Figure S1: Structure of (R)-Methyloxirane

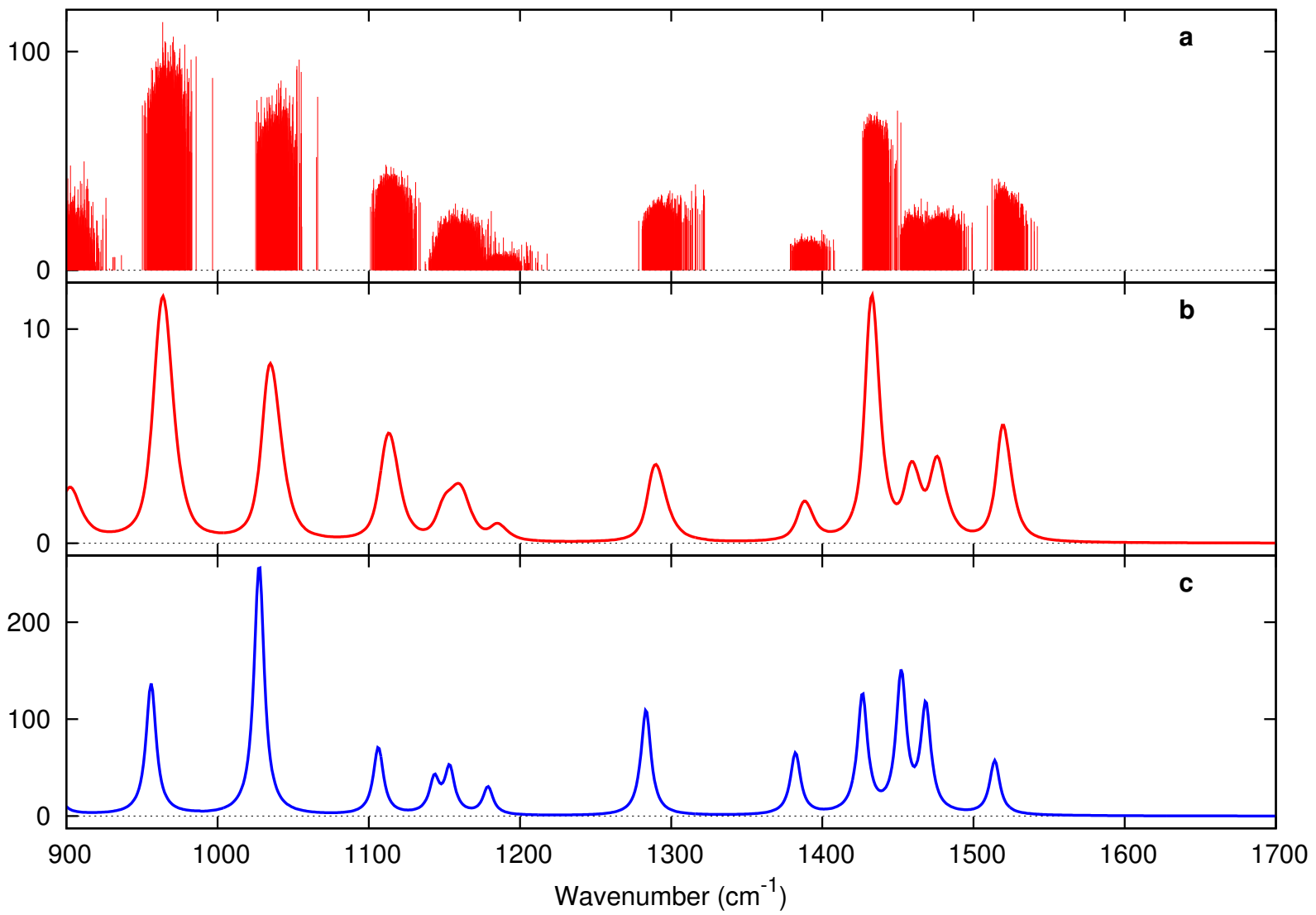

Figure S2: IR spectrum of (R)-Methyloxirane in aqueous solution. Intensity are arbitrary. (a) Stick spectrum described by using QM/FQ/PCM model. 2000 snapshots considered. (b) Convoluted spectrum described by means of QM/FQ/PCM model. A FWHM of 4 $\mathrm{cm}^{-1}$ for each snapshot is considered. (c) Spectrum obtained by using QM/PCM model. A FWHM of $10 \mathrm{~cm}^{-1}$ is considered. 

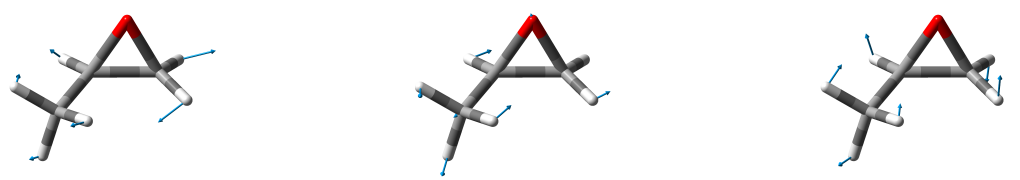

$905.84 \mathrm{~cm}^{-1}$

$954.83 \mathrm{~cm}^{-1}$

$1039.80 \mathrm{~cm}^{-1}$
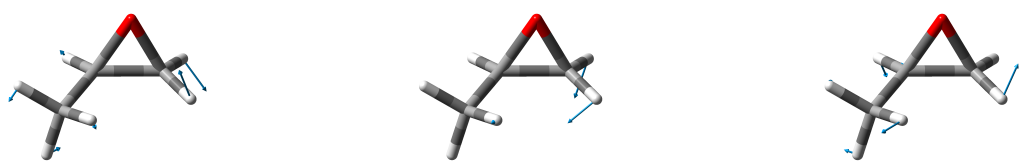

$1111.33 \mathrm{~cm}^{-1}$

$1160.14 \mathrm{~cm}^{-1}$

$1163.76 \mathrm{~cm}^{-1}$
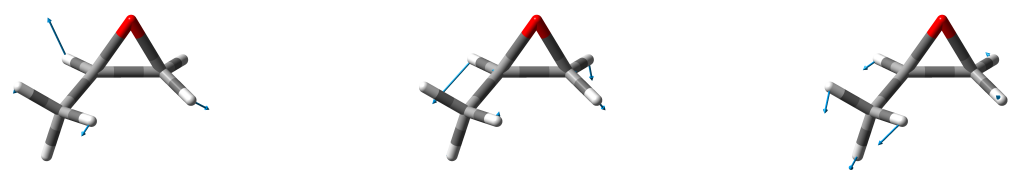

$1199.00 \mathrm{~cm}^{-1}$

$1292.60 \mathrm{~cm}^{-1}$

$1387.30 \mathrm{~cm}^{-1}$

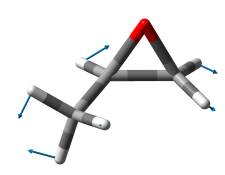

$1431.57 \mathrm{~cm}^{-1}$

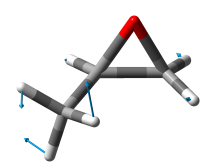

$1462.80 \mathrm{~cm}^{-1}$

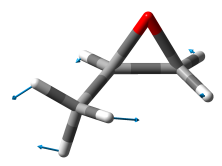

$1477.03 \mathrm{~cm}^{-1}$

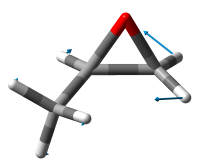

$1521.07 \mathrm{~cm}^{-1}$

Figure S3: Normal modes of (R)-Methyloxirane with QM/FQ/PCM model. The frequency of each mode is reported in parentheses. These modes are referred to one random snapshot (1445). 


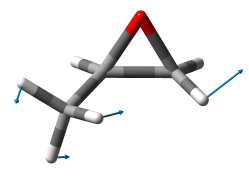

$895.66 \mathrm{~cm}^{-1}$

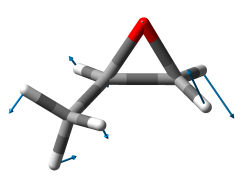

$1106.46 \mathrm{~cm}^{-1}$

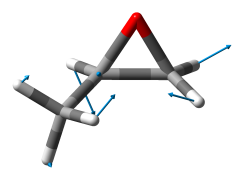

$1177.15 \mathrm{~cm}^{-1}$

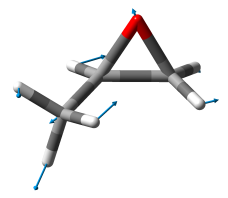

$956.33 \mathrm{~cm}^{-1}$

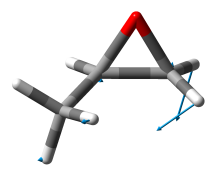

$1142.33 \mathrm{~cm}^{-1}$

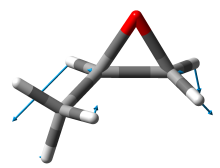

$1283.78 \mathrm{~cm}^{-1}$

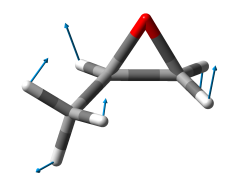

$1026.91 \mathrm{~cm}^{-1}$

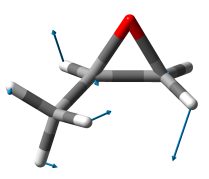

$1152.80 \mathrm{~cm}^{-1}$

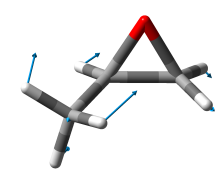

$1380.56 \mathrm{~cm}^{-1}$

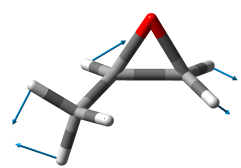

$1424.28 \mathrm{~cm}^{-1}$

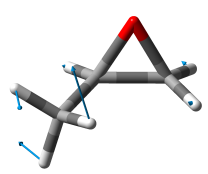

$1446.49 \mathrm{~cm}^{-1}$

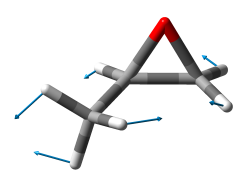

$1463.31 \mathrm{~cm}^{-1}$

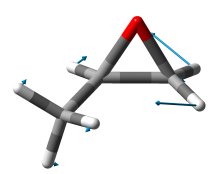

$1511.54 \mathrm{~cm}^{-1}$

Figure S4: Normal modes of (R)-Methyloxirane with QM/PCM model. The frequency of each mode is reported in parentheses. 


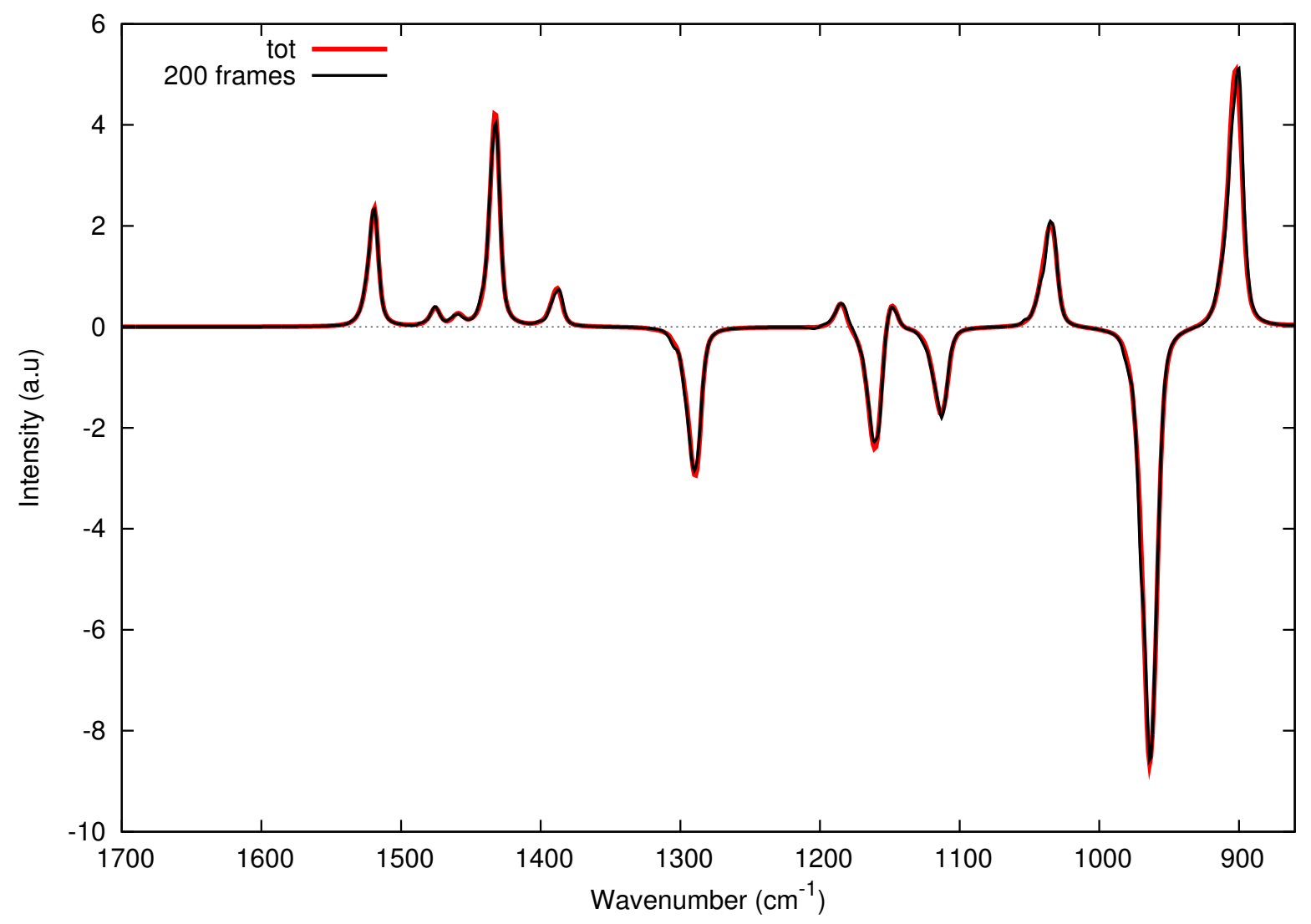

Figure S5: Calculated averaged QM/FQ/PCM VCD spectrum obtained by averaging over 2000 snapshots (red) or 200 (black) snapshots chosen in a regular way (1 over 10). 


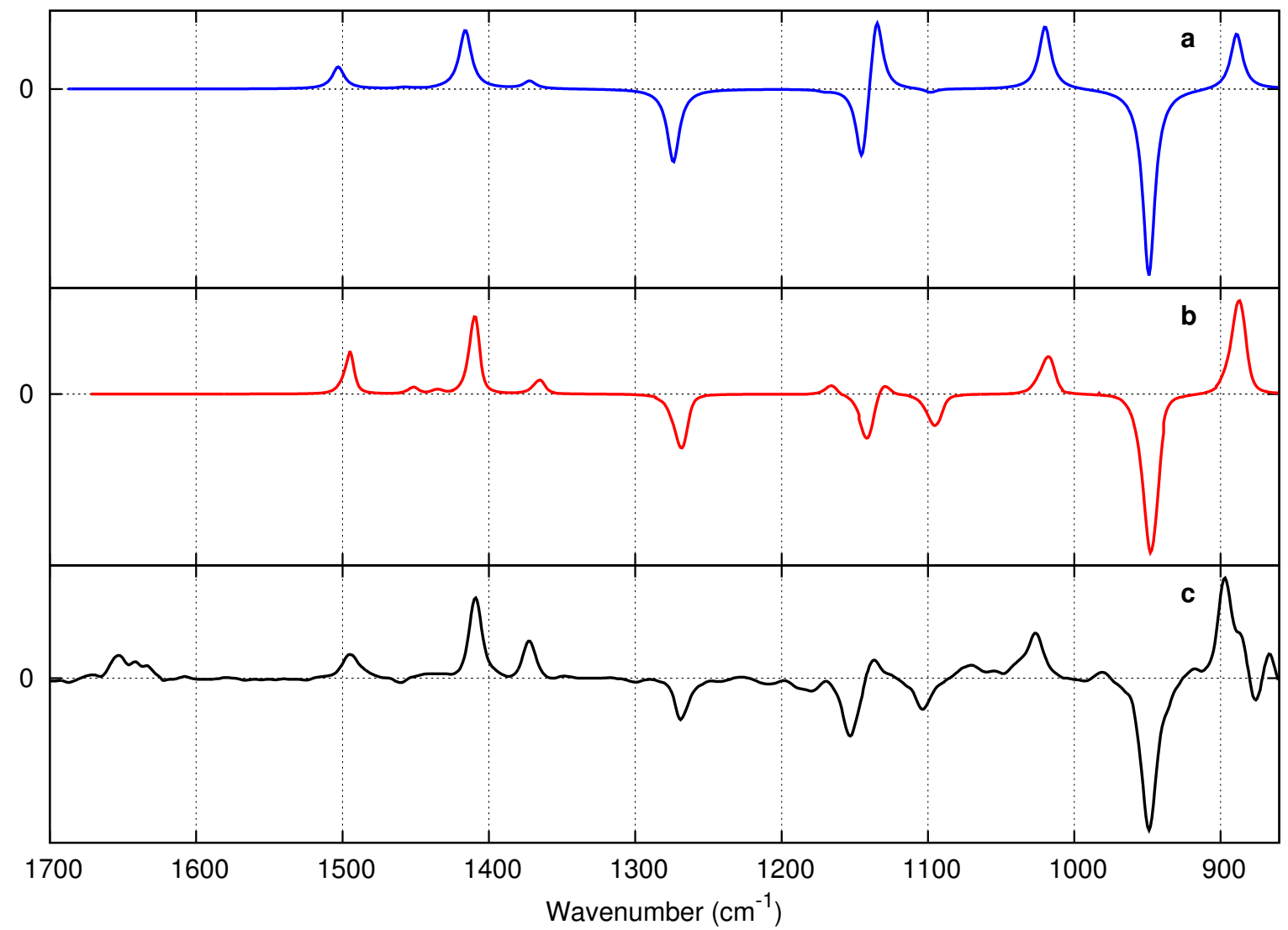

Figure S6: (a) PCM, (b) QM/FQ/PCM and (c) experimental ${ }^{1}$ VCD spectra of (R)methyloxirane in aqueous solution. All spectral intensities and the frequencies are scaled so that the peaks at about $960 \mathrm{~cm}^{-1}$ have the same intensity and energy. 


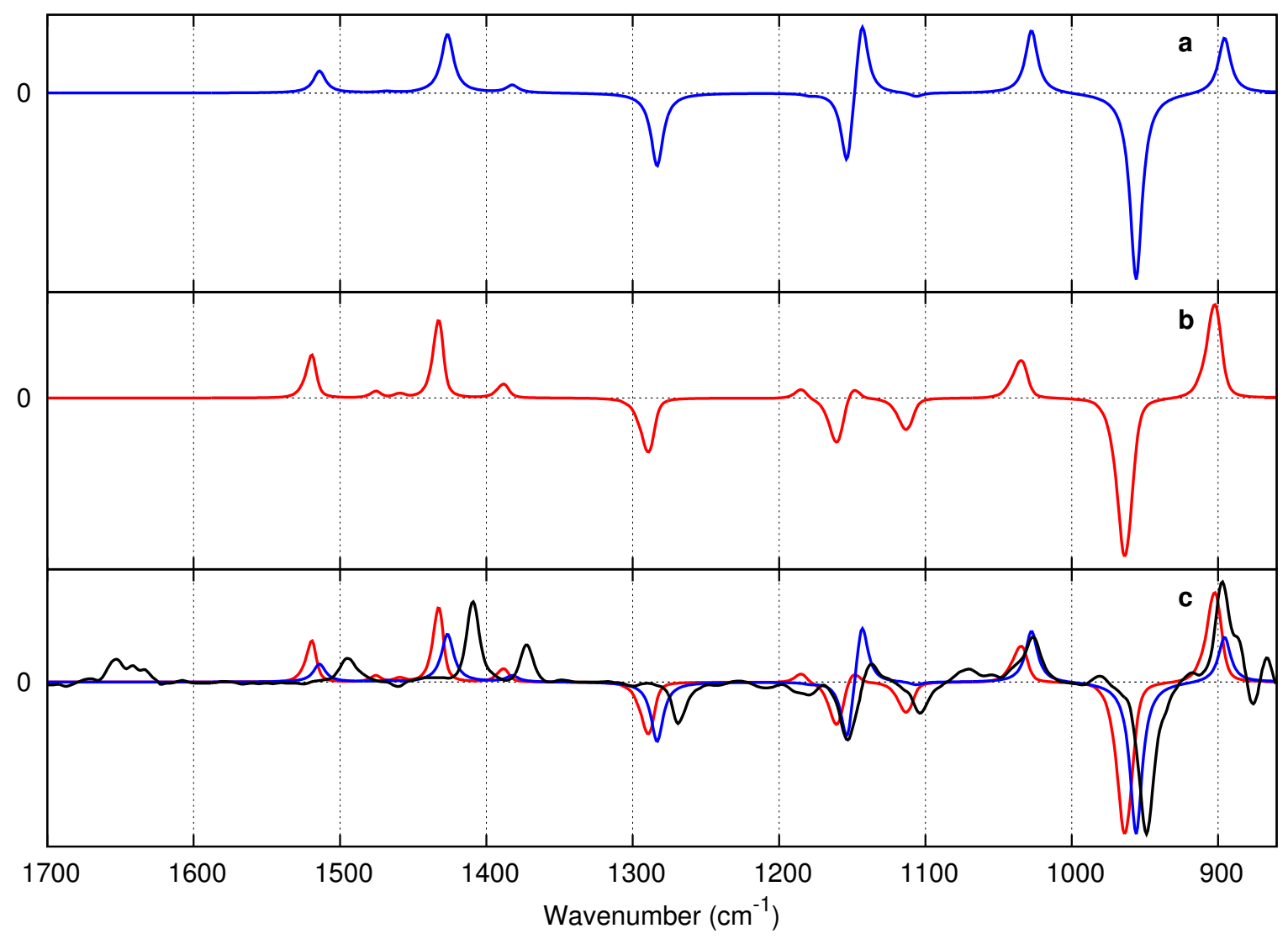

Figure S7: Calculated non-scaled VCD spectra of (R)-Methyloxirane in aqueous solution. (a) QM/PCM model. (b) QM/FQ/PCM model. (c) Comparison between QM/PCM (blue), QM/FQ/PCM model (red) and the experimental spectrum ${ }^{1}$ (black). All spectral intensities are scaled so that the peaks at about $960 \mathrm{~cm}^{-1}$ have the same intensity. Intensities are given in arbitrary units. 


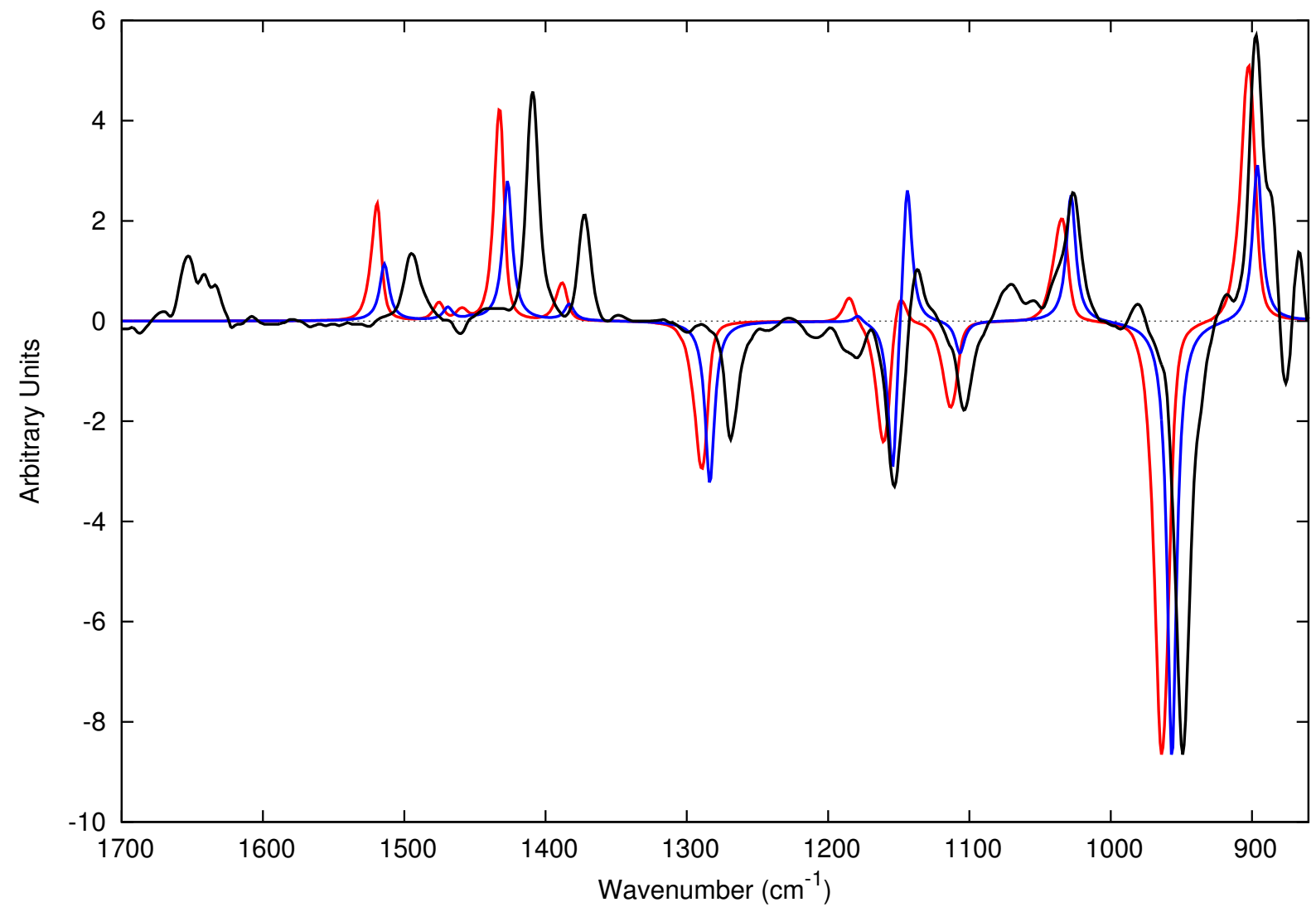

Figure S8: (R)-Methyloxirane in aqueous solution. Comparison between calculated QM/PCM VCD spectrum accounting for cavity field and nonequilibrium effects ${ }^{2-4}$ (blue), averaged $\mathrm{QM} / \mathrm{FQ} / \mathrm{PCM} \mathrm{VCD}$ spectrum (red) and experimental data (black). Intensities are given in arbitrary units. Wavenumbers are not scaled. 


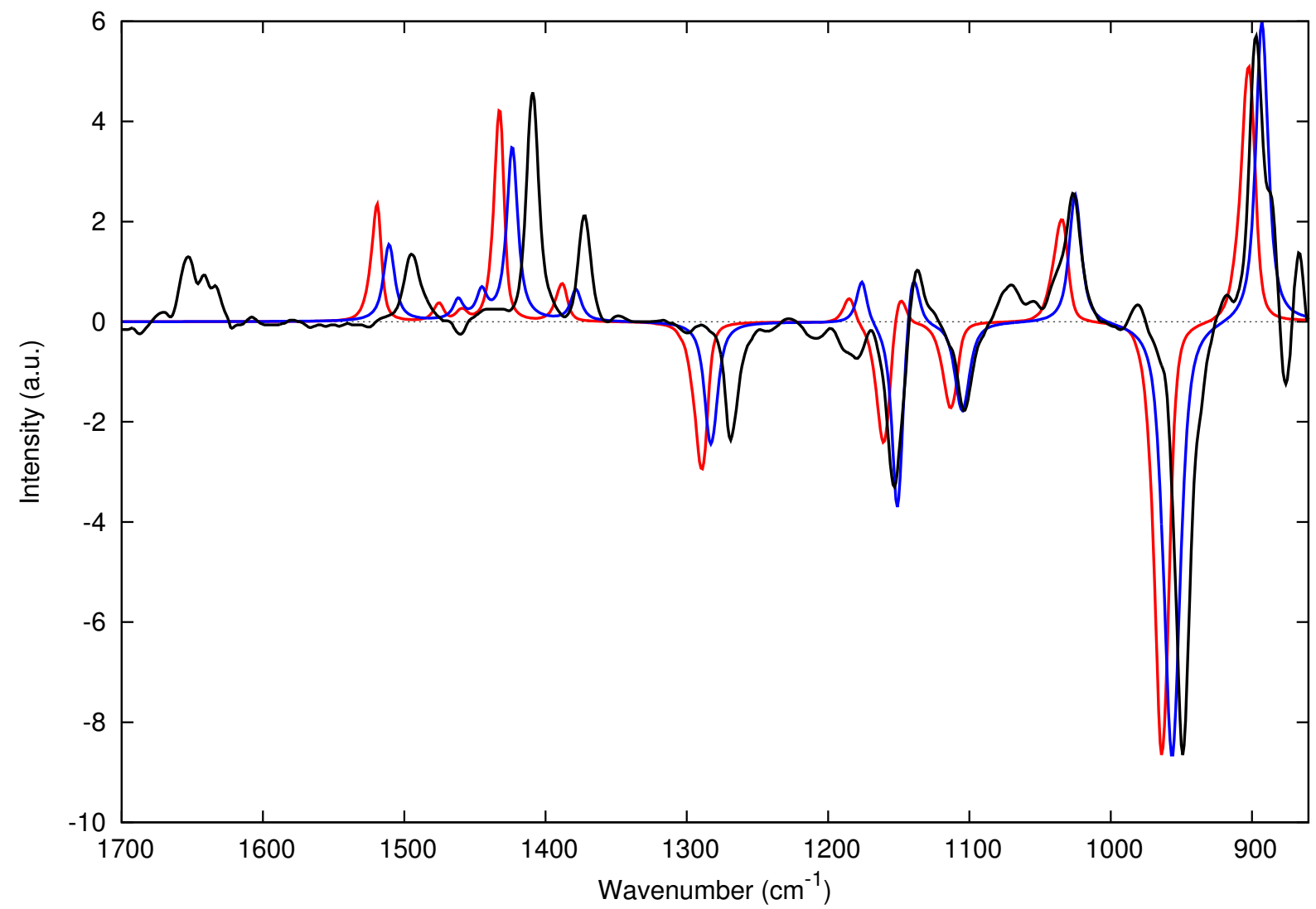

Figure S9: (R)-Methyloxirane in aqueous solution. Comparison between averaged QM/PCM VCD spectrum obtained by applying the PCM to optimized QM/FQ/PCM structures (blue), averaged $\mathrm{QM} / \mathrm{FQ} / \mathrm{PCM} \mathrm{VCD}$ spectrum (red) and experimental data (black). Intensities are given in arbitrary units. Wavenumbers are not scaled. 


\section{S2 (L)-Alanine}

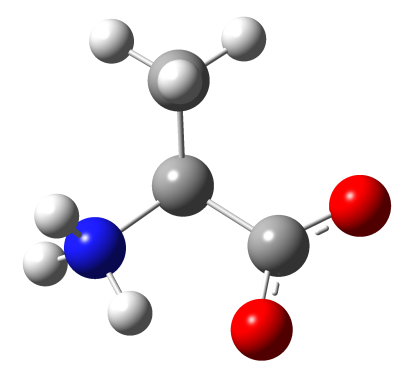

Figure S10: Structure of (L)-Alanine: zwitterionic form, the most stable one at $\mathrm{pH}=7$

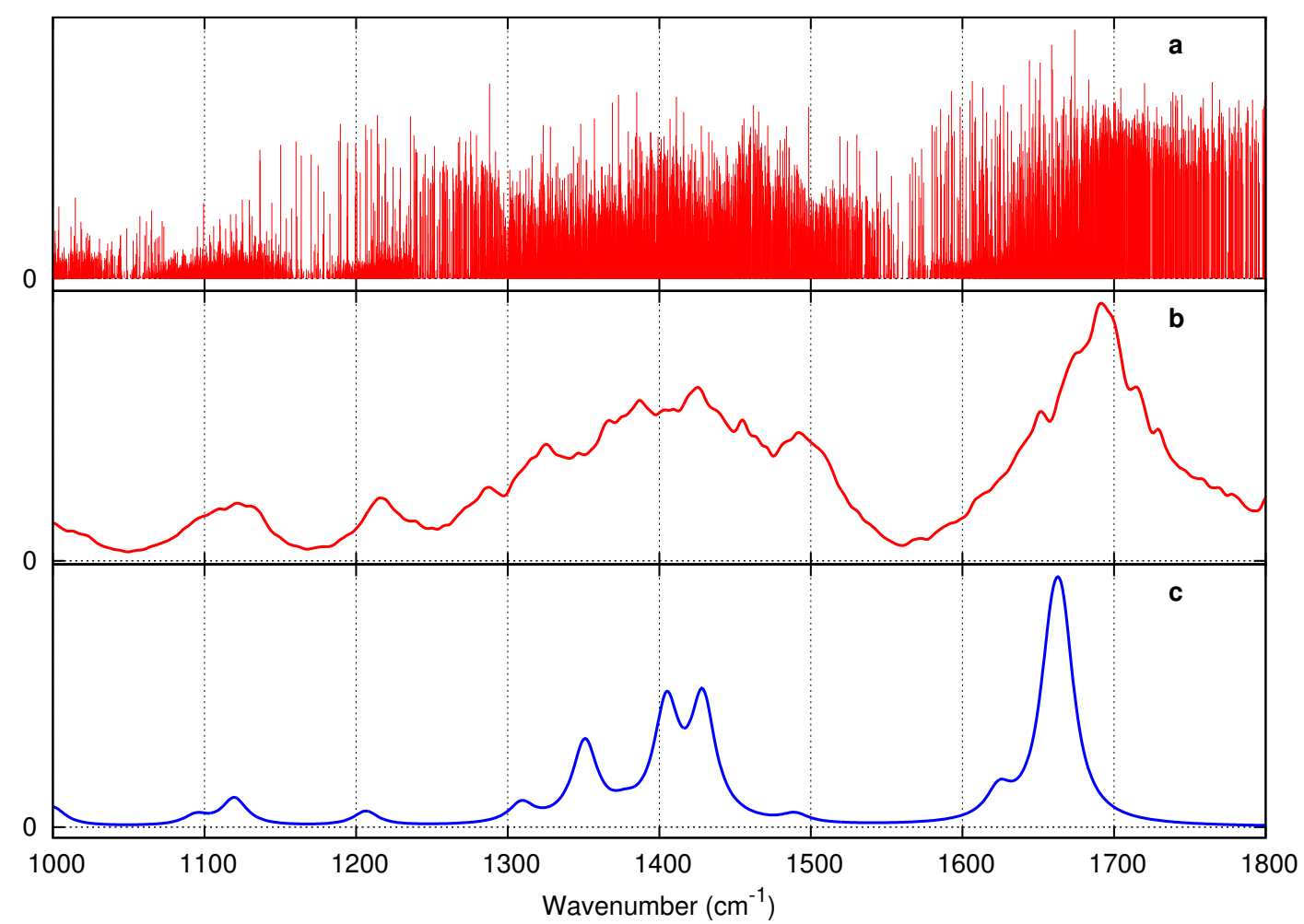

Figure S11: IR spectrum of (L)-Alanine in aqueous solution. Intensities are arbitrary. (a) Stick spectrum described by using QM/FQ/PCM model. 1000 snapshots considered. (b) Convoluted spectrum described by means of QM/FQ/PCM model. A FWHM of $4 \mathrm{~cm}^{-1}$ for each snapshot is considered. (c) Spectrum obtained by using QM/PCM model. A FWHM of $10 \mathrm{~cm}^{-1}$ is considered. 


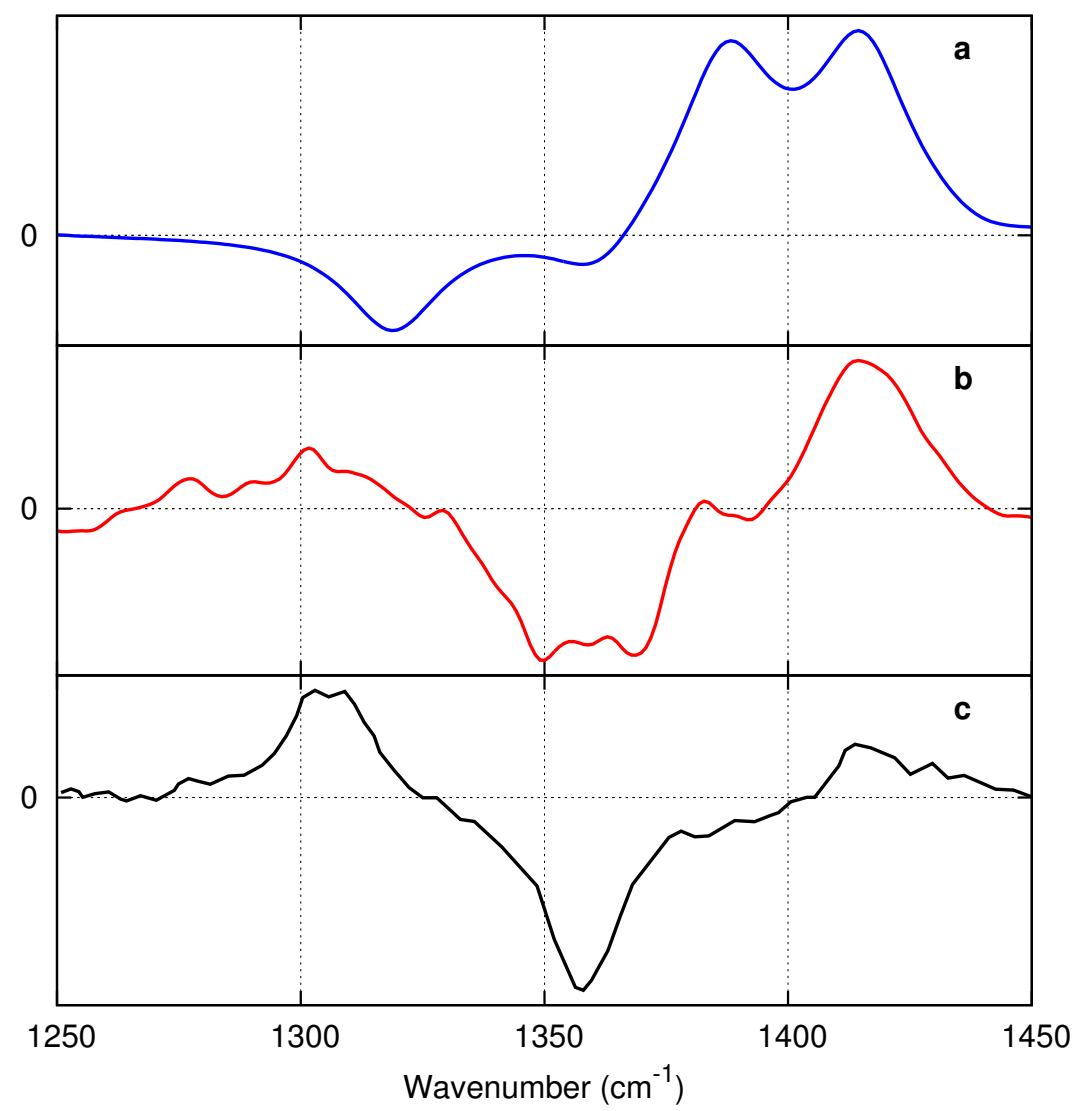

Figure S12: (a) PCM, (b) QM/FQ/PCM and (c) experimental ${ }^{5}$ VCD spectra of (L)-alanine in aqueous solution. All spectral intensities and the frequencies are scaled so that the peaks at about $1360 \mathrm{~cm}^{-1}$ have the same intensity and energy. 


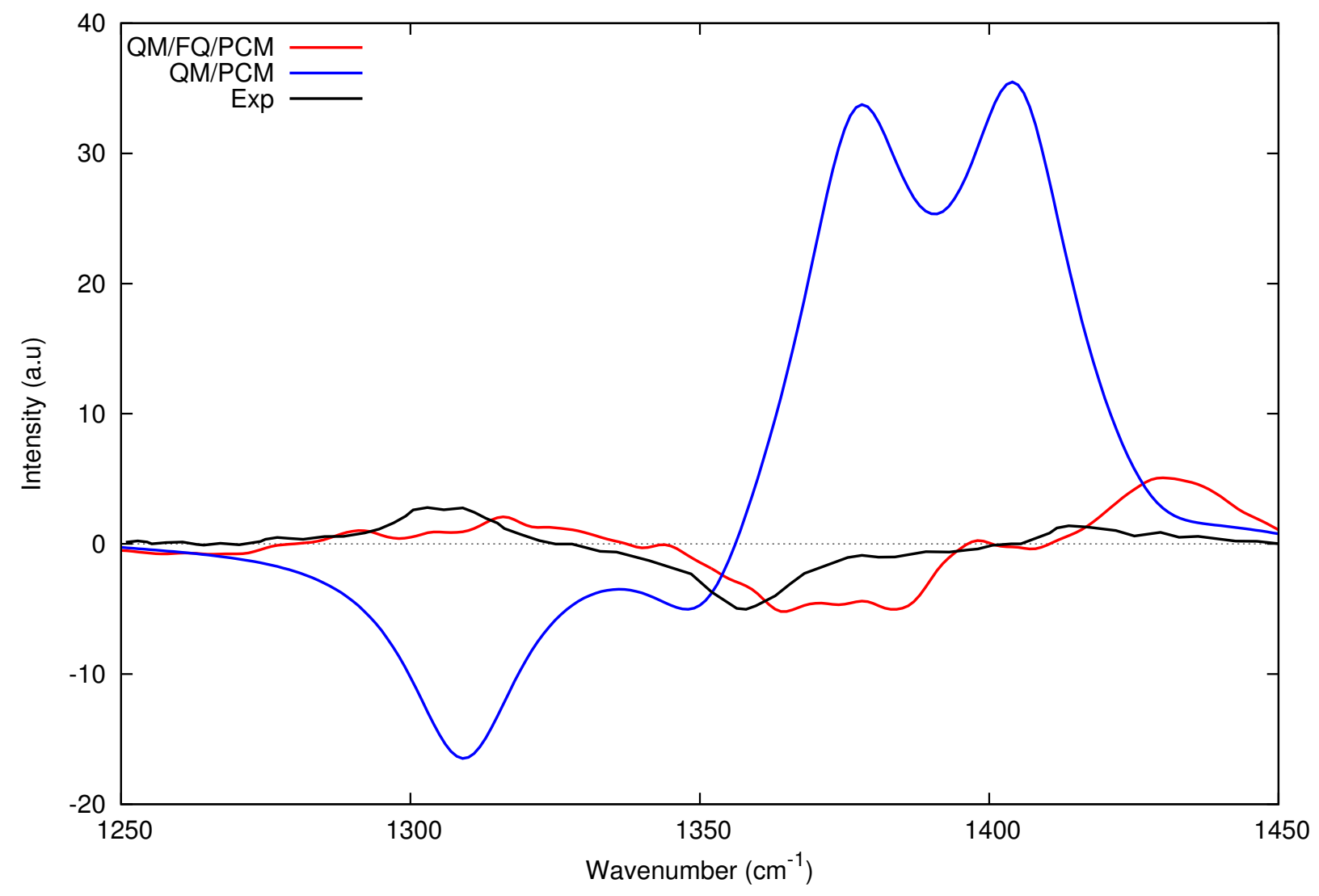

Figure S13: VCD spectrum of (L)-Alanine in aqueous solution. Intensities are arbitrary. (a) QM/PCM model. (b) QM/FQ/PCM model. (c) Comparison between QM/PCM (blue), QM/FQ/PCM model (red) and experimental spectrum (black). ${ }^{5}$ Wavenumbers are not scaled. Intensities are scaled so that the peak at about $1360 \mathrm{~cm}^{-1}$ has the same high. 


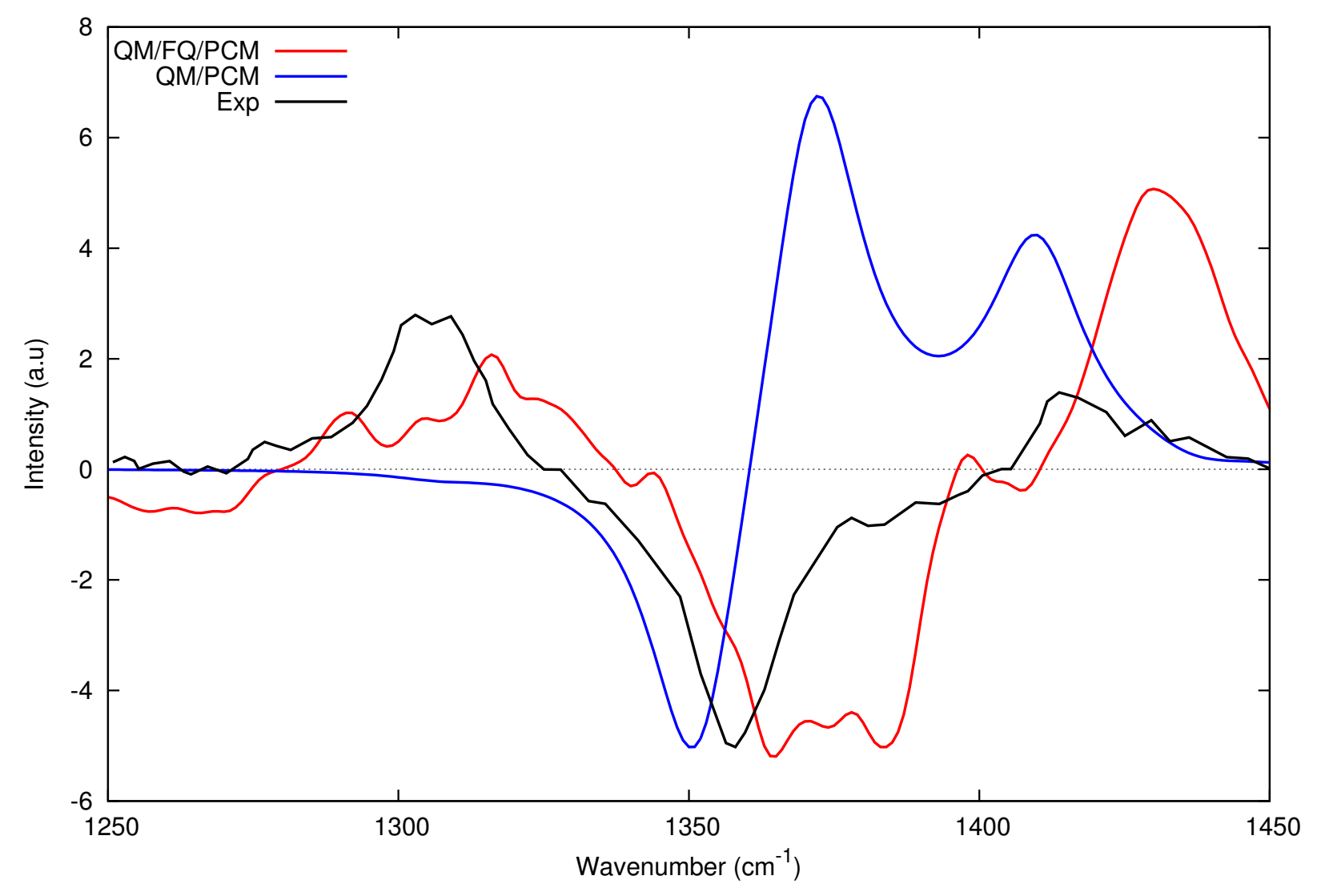

Figure S14: Effects of Local Field and Vibrational Nonequilibrium in PCM model: VCD spectrum of (L)-Alanine in aqueous solution. Intensities are arbitrary. Comparison between $\mathrm{QM} / \mathrm{PCM}$ (blue), QM/FQ/PCM (red) and experimental data ${ }^{5}$ (black). Wavenumbers are not scaled. Intensities are scaled so that the peak at about $1360 \mathrm{~cm}^{-1}$ has the same high. 


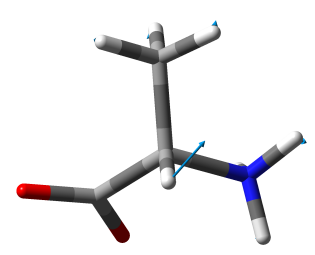

$1320.48 \mathrm{~cm}^{-1}$

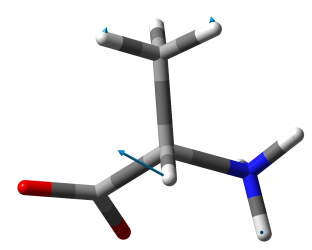

$1384.64 \mathrm{~cm}^{-1}$

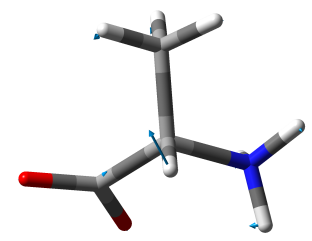

$1359.06 \mathrm{~cm}^{-1}$

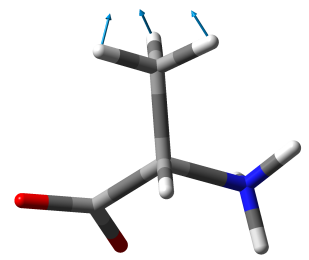

$1429.01 \mathrm{~cm}^{-1}$

Figure S15: Normal modes of (L)-Alanine with QM/FQ/PCM model. The frequency of each mode is reported in parentheses. These modes are referred to one random snapshot (565). 

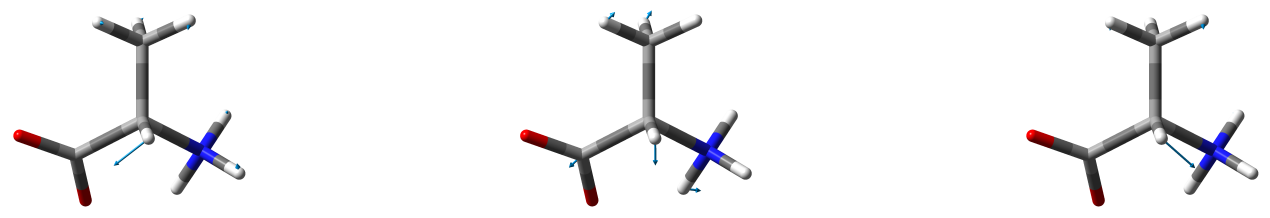

$1309.19 \mathrm{~cm}^{-1}$

$1350.98 \mathrm{~cm}^{-1}$

$1377.03 \mathrm{~cm}^{-1}$
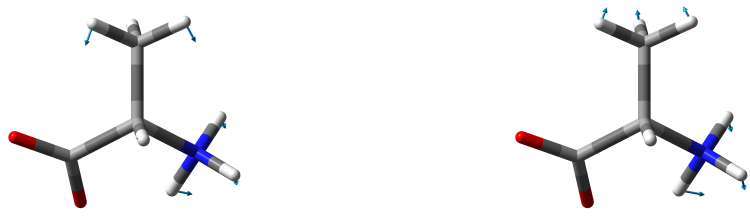

$1404.77 \mathrm{~cm}^{-1}$

$1428.70 \mathrm{~cm}^{-1}$

Figure S16: Normal modes of (L)-Alanine with QM/PCM model. The frequency of each mode is reported in parentheses. 


\section{References}

(1) Losada, M.; Nguyen, P.; Xu, Y. J. Phys. Chem A 2008, 112, 5621-5627.

(2) Cappelli, C.; Corni, S.; Mennucci, B.; Cammi, R.; Tomasi, J. J. Phys. Chem A 2002, 106, $12331-12339$.

(3) Cappelli, C.; Lipparini, F.; Bloino, J.; Barone, V. J. Chem. Phys. 2011, 135, 104505.

(4) Cappelli, C.; Corni, S.; Cammi, R.; Mennucci, B.; Tomasi, J. J. Chem. Phys. 2000, 113, $11270-11279$.

(5) Diem, M. J. Am. Chem. Soc. 1988, 110, 6967-6970. 